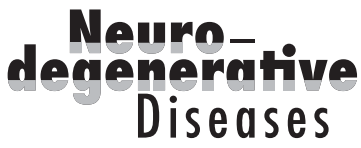

\title{
APOE-Mimetic Peptides Reduce Behavioral Deficits, Plaques and Tangles in Alzheimer's Disease Transgenics
}

\author{
M.P. Vitek ${ }^{a, b}$ D.J.Christensen ${ }^{a} \quad$ D. Wilcock ${ }^{b} \quad$ J. Davis ${ }^{c}$ W.E. Van Nostrand ${ }^{c}$ \\ F.Q. Li ${ }^{a}$ C.A. Colton ${ }^{b}$ \\ ${ }^{a}$ Cognosci, Inc., Research Triangle Park, N.C., ${ }^{b}$ Duke University Medical Center, Durham, N.C., and \\ 'Stony Brook University, Stony Brook, N.Y., USA
}

\section{Key Words}

Apolipoprotein $\mathrm{E} \cdot$ Neuroprotection - Anti-inflammatory activity $\cdot \mathrm{SET}\left(\right.$ or $\left.\mathrm{I}_{2}^{\mathrm{PP} 2 \mathrm{~A}}\right) \cdot$ Protein phosphatase $2 \mathrm{~A}$.

Dephosphorylation $\cdot$ Neurofibrillary tangles $\cdot$ Tau •

Amyloid $\cdot$ Amyloid- $\beta$ peptide $\cdot$ Alzheimer's disease

\begin{abstract}
Background: After age, the second largest risk factor for Alzheimer's disease (AD) is apolipoprotein E (APOE) genotype, where APOE4 is associated with lower apoE protein levels, more severer brain pathology, enhanced inflammation and disease. Small peptides corresponding to the receptorbinding region of apoE mimic the anti-inflammatory activity of the apoE holoprotein. These apoE mimetics greatly improve behavioral outcomes and neuronal survival in head trauma models that display AD pathology and neuronal loss. Objective: To determine whether apoE mimetics change behavior, inflammation and pathology in CVND-AD (SwDI-APP/ $\mathrm{NOS2}^{\left.-{ }^{-}\right)}$) transgenic mice. Methods: Starting at 9 months, apoE peptides were subcutaneously administered 3 times per week for 3 months followed by behavioral, histochemical and biochemical testing. Results: Treatment with apoE mimetics significantly improved behavior while decreasing
\end{abstract}

the inflammatory cytokine IL-6, neurofibrillary tangle-like and amyloid plaque-like structures. Biochemical measures matched the visible pathological results. Conclusions: Treatment with apoE mimetics significantly improved behavior, reduced inflammation and reduced pathology in CVND-AD mice. These improvements are associated with apoE-mimetic-mediated increases in protein phosphatase $2 \mathrm{~A}$ activity. Testing in additional AD models showed similar benefits, reinforcing this novel mechanism of action of apoE mimetics. These data suggest that the combination of anti-inflammatory and neuroprotective activities of apoE mimetics represents a new generation of potential therapeutics for $A D$.

Copyright $\odot 2012$ S. Karger AG, Basel

Since the discovery that apolipoprotein E4 (APOE4) carriers have increased susceptibility for developing Alzheimer's disease (AD) [1-4], the precise mechanisms causing this association have remained undefined. To begin to address this issue, we created small peptide mimetics of apoE that derive from its receptor-binding domain and studied their biological activities. ApoE133-149 (or COG133) is a 17-amino-acid peptide that displays antiinflammatory and neuroprotective activities in a variety

\section{KARGER \\ Fax +41613061234 \\ E-Mail karger@karger.ch}

www.karger.com
(C) 2012 S. Karger AG, Basel

$1660-2854 / 12 / 0104-0122 \$ 38.00 / 0$

Accessible online at:

www.karger.com/ndd
M.P. Vitek

Cognosci, Inc.

79 T W Alexander Drive, PO Box 110606

Research Triangle Park, NC 27709 (USA)

Tel. +1 919765 0028, E-Mail mikevitek@ cognosci.com 


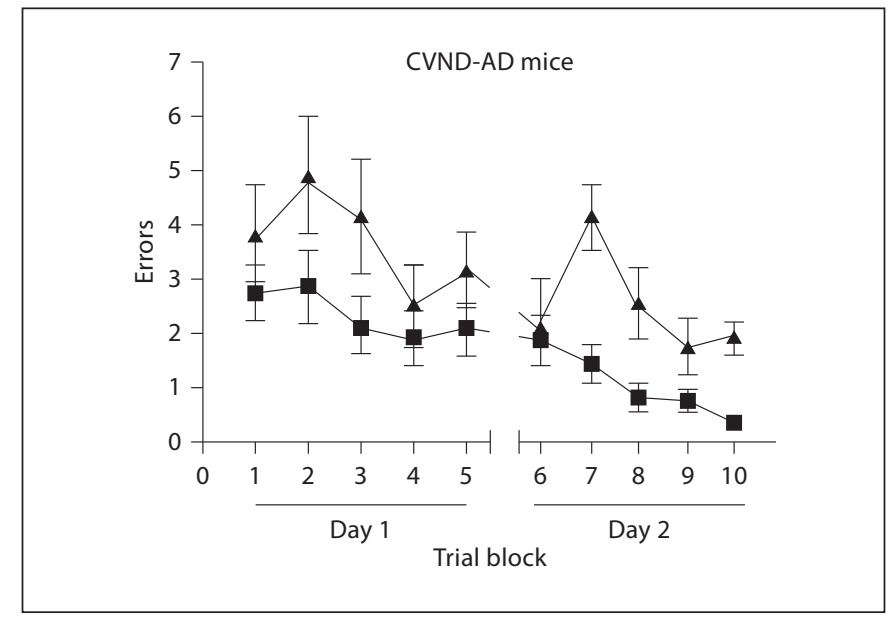

Fig. 1. Behavioral performance on the 2-day RAWM is significantly improved in COG1410-treated CVND-AD mice compared to LRB controls. Mice ( $\mathrm{n}=8 /$ group) were treated with $4 \mathrm{mg} / \mathrm{kg}$ COG1410 (squares) or LRB (triangles) by subcutaneous injection 3 times per week during months 9,10 and 11 of life. In month 12, RAWM testing was performed as per Wilcock et al. [3]. $\mathrm{p}<0.05$ by 2-way ANOVA for errors, trial block and interaction (Prism, GraphPad, Inc.).

of cell-based and whole animal models [2, 5]. Analogs of apoE133-149 include COG1410, a 12-amino-acid peptide that is also active in animal models, but with increased potency and extended latency between injury and time to treatment $[1,6]$.

We defined one mechanism of apoE action by showing that COG133 and the apoE holoprotein specifically bind the SET protein, which is also known as inhibitor 2 of protein phosphatase 2A (I2PP2A) [1]. COG133/ApoEmediated inhibition of SET leads to a derepression and re-activation of PP2A so that PP2A-mediated phosphatase activity levels increase [1]. Indeed, we found that subcutaneous administration of COG1410 resulted in increased PP2A activity in the brains of mice [1]. Wang et al. [7] showed that overexpression of SET generated phosphorylated tau containing neurofibrillary tangle (NFT)-like structures and neuronal loss in the brain. Similarly, Deters et al. [8] showed that expression of mutated PP2A also led to accumulation of phosphorylated tau/NFT-like structures in transgenic mice. Since SET is overexpressed and $\mathrm{PP} 2 \mathrm{~A}$ is repressed in $\mathrm{AD}$ brains, we hypothesized that the addition of apoE-mimetic peptides will increase PP2A phosphatase activity, reduce inflammation, reduce phosphorylated tau and reduce neuron loss.

APOE Mimetics and Behavioral Deficits, Plaques and Tangles in AD Transgenics
To test the idea that stimulating PP2A could reduce pathology and improve outcomes, we treated SwDI-APP/ $\mathrm{NOS}^{-l-}$ (CVND-AD) mice with COG1410 followed by assessment of behavioral performance, cytokines and AD-like pathology (amyloid plaques and NFTs). The CVND-AD strain expresses 3 human APP mutations (Swedish K760N/M671L, Dutch E693Q and Iowa D694N) on an $\mathrm{mNOS}^{-/-}$background [3]. These mutations promote increased levels of $A \beta 1-40$ versus $A \beta 1-42$, and direct amyloid deposition to the cerebrovasculature [9]. NO levels are reduced to reflect the lower NO levels produced by human versus mouse NOS2 activity during an immune response, thus restricting its bioavailability as an antioxidant (among many other survival functions) [10]. The resultant double-transgenic strain demonstrates amyloid deposition; mouse tau hyperphosphorylation, aggregation and redistribution; neuronal loss and cognitive defects that together strongly mimic the behavior and pathology found in humans with $\mathrm{AD}$ [3]. We treated CVND$\mathrm{AD}$ mice with COG1410 at $4 \mathrm{mg} / \mathrm{kg}$ or lactated Ringer's buffer (LRB) by subcutaneous administration 3 times per week for 3 months beginning when mice were 9 months of age. No toxic effects of this treatment were observed, which was followed by behavioral assessment using the 2-day radial arm water maze (RAWM) [3]. As shown in figure 1, both COG1410 and vehicle-treated controls learned the task in the early trials followed by a progressive improvement in the COG1410 animals that was not observed in the controls. This learning and memory was durable, as the COG1410-treated animals retained good performance on day 2 of testing, while controls did not. Rotorod performance was not different between the groups, suggesting that altered motor function could not account for the RAWM results (data not shown).

Following behavioral testing, animals were lethally anesthetized, perfused by cardiac puncture and brains harvested for analysis of pathologies using standard immunocytochemical and biochemical methods. Consistent with NFT-like pathology seen in untreated CVND$\mathrm{AD}$ mice [3], numerous AT8-positive, phosphorylated tau-containing structures can be seen in vehicle-treated subiculum and cortex (fig. 2). These hyperphosphorylated tau deposits composed of normal mouse tau (not mutated human tau) are observed in the cortex and in cells surrounding blood vessels in the subiculum, and are quite similar to those typically found in patients with $\mathrm{AD}$ $[11,12]$. We previously reported double-label immunofluorescence where neuron-specific $\beta$-tubulin antibodies labeled cells that were also AT8-positive thereby identifying phosphorylated mouse tau in cortical neurons [3]. 
Fig. 2. AT8 staining for phosphorylated mouse tau in the brains of CVND-AD mice treated with COG1410 is visually reduced compared to LRB vehicle. a, c Treated with LRB. b, d Treated with COG1410. Treatments were by subcutaneous injection 3 times per week for months 9, 10 and 11 , followed by perfusion, fixation in $4 \%$ paraformaldehyde, cutting of frozen sections and staining of free-floating sections with AT8. Representative pictures from individual mice are shown.

Fig. 3. $6 \mathrm{E} 10$ staining of $A \beta$ in the cortex of CVND-AD mice treated with vehicle (a) is visually reduced after COG1410 treatment (b) by subcutaneous administration 3 times per week for 3 months as was confirmed by Western blotting. Representative pictures from individual mice are shown.
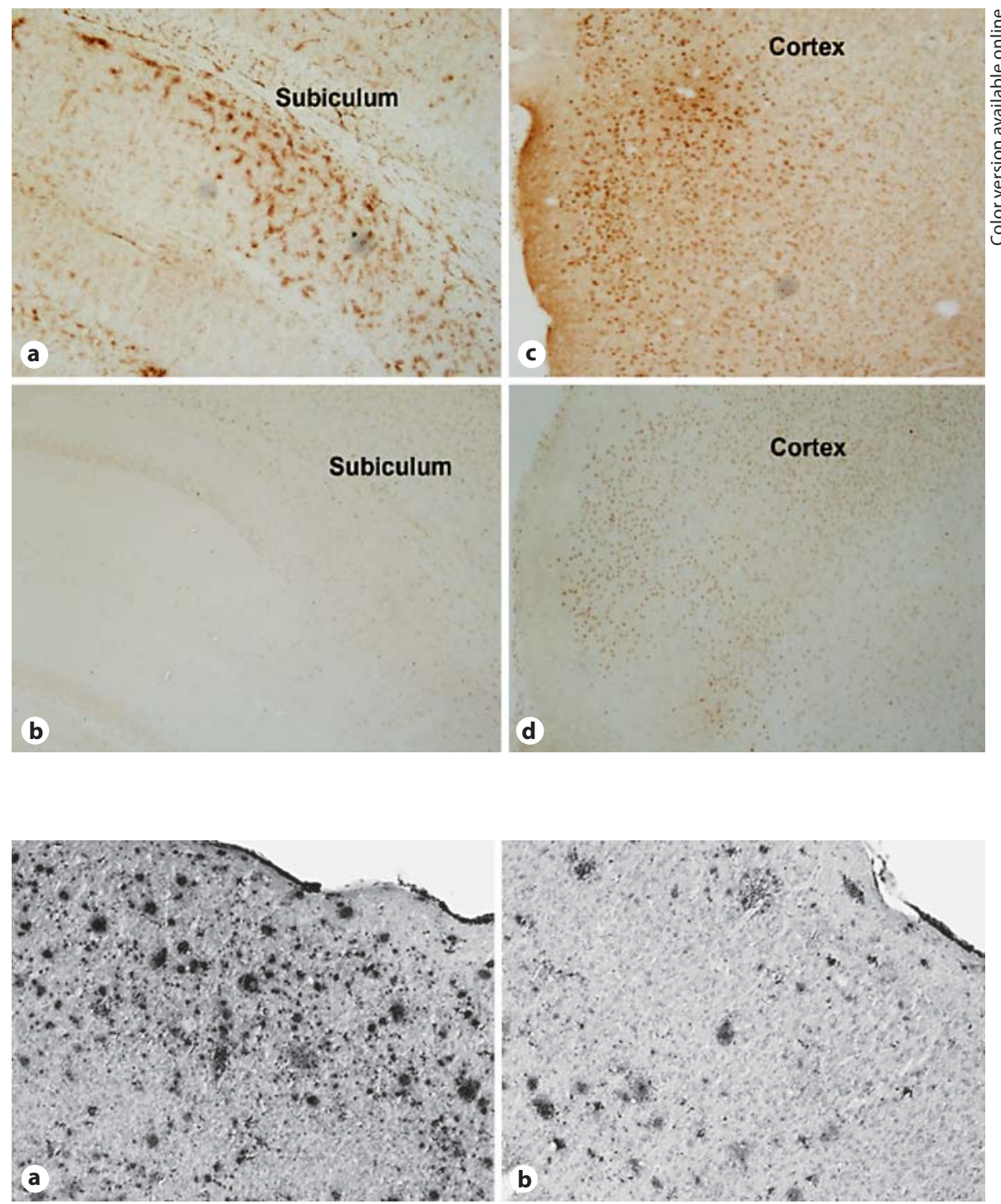

Similarly, we previously reported double-immunofluorescent staining where NeuN antibodies and AT8 antibodies identified phosphorylated tau in perivascular neurons of CVND-AD mice and of humans with AD [13]. In this same study, we found that microglia, but not astrocytes, also stained for AT8-positive phosphorylated mouse tau [13]. In contrast to vehicle, AT8-positive cells were visibly reduced in the COG1410-treated cortex and subiculum (fig. 2).

Consistent with amyloid plaque-like pathology seen in untreated CVND-AD mice [3], extensive 6E10-positive, $A \beta$-containing structures are seen in vehicle-treated cortex, which are greatly reduced in the COG1410-treated cortex (fig. 3). Similar results were observed in the hip- pocampus (data not shown). To confirm the visual reduction in the plaque burden, Western blots of brain lysates showed that total $A \beta$ levels relative to $\beta$-actin levels were $0.7 \pm 0.21$ units in COG1410-treated $(\mathrm{n}=4)$ mice, which is significantly less than $1.5 \pm 0.25$ units in controls ( $\mathrm{n}=$ 4) (t test, $p<0.05$; blots not shown).

Neuroinflammation is also thought to contribute to both AD pathology and behavioral deficits. Since COG1410 is an effective anti-inflammatory agent, we measured selected cytokines in the brain by quantitative RT-PCR using wild-type mouse brains as a calibrator [14] and primers from Applied BioSystems (Carlsbad, Calif., USA). We found that IL- 6 mRNA levels were significantly reduced in the brains of COG1410-treated animals, 


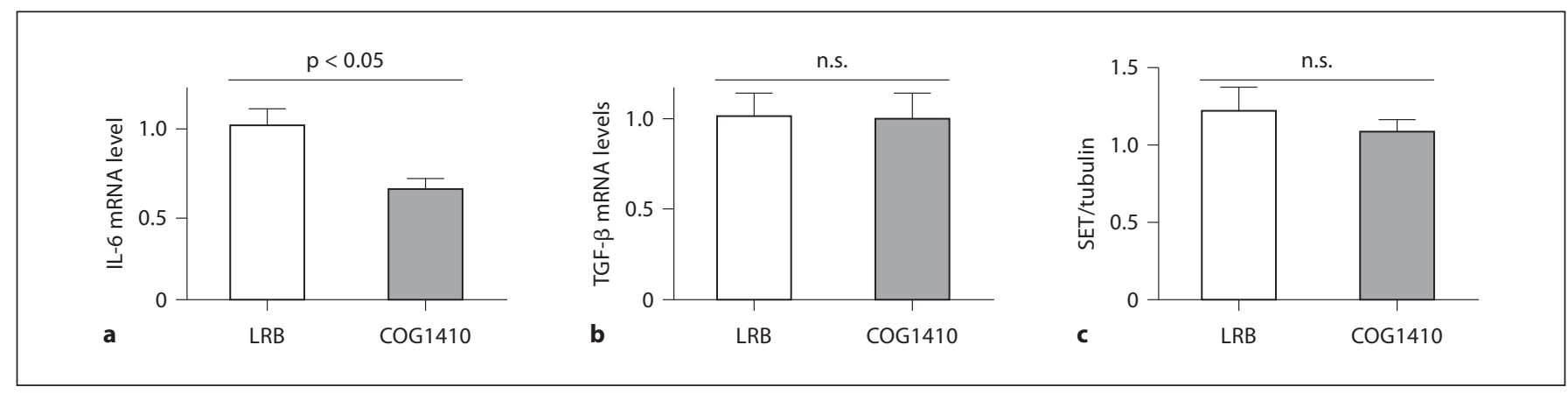

Fig. 4. a IL-6 mRNA levels from the brains of CVND-AD mice treated with COG1410 are significantly less than from those treated with vehicle (LRB). Quantitative RT-PCR was performed with primers specific for IL- 6 and actin (as an internal standard) using wild-type untreated mouse brain as a calibrator. $\mathrm{n}=4$ mice per group. $\mathrm{p}<0.05$. $\mathbf{b}$ TGF- $\beta$ mRNA levels from the brains of CVND-AD mice treated with COG1410 are not significantly different than from those treated with vehicle (LRB). Quantitative RT-PCR was performed with primers specific for TGF- $\beta$ and ac-

while levels of TGF- $\beta$ were not different between vehicle and COG1410 treatments (fig. 4a, b). Since COG1410 antagonizes SET, which could affect SET levels, we also measured SET protein levels by Western blotting of whole brain lysates and densitometric scanning of immunoreactive SET protein bands. As shown in figure 4c, we found no statistical difference in SET levels between vehicle and COG1410-treated animals.

We have tested COG1410, a small peptide mimetic of holo-apoE, for its effect on $\mathrm{AD}$ pathology in several transgenic models. In this short report, treatment of CVND-AD mice with COG1410 showed significant reductions in NFT-like and amyloid plaque-like brain pathologies by immunocytochemistry. The observed visual reduction in $A \beta$ immunocytochemical staining was confirmed by quantitative Western blotting for total $A \beta$ levels from control and COG1410-treated mouse brains. These reductions in phosphorylated tau and $A \beta$ levels directly match earlier work in cell cultures transfected with PP2A constructs that overexpress and increase PP2A-mediated phosphatase activity [15]. Reductions in phosphorylated tau were also observed by van Eersel et al. [16] using sodium selenate to increase PP2A activity. On the other side of the SET/PP2A complex, Wang et al. [7] showed that increasing SET levels led to increased phosphorylated tau deposits in the brain. We found that brain levels of SET protein did not change with 12 weeks of vehicle or COG1410 treatment, suggesting that COG1410-mediated antagonism of SET, and not a change tin (as an internal standard) using wild-type untreated mouse brain as a calibrator. $\mathrm{n}=4$ mice per group. $\mathrm{p}>0.05$. $\mathrm{c}$ SET protein levels from the brains of CVND-AD mice treated with COG1410 are similar to those treated with vehicle (LRB). Whole brain lysates were Western blotted and probed with anti-SET and antitubulin antibodies. Immunoreactive bands corresponding to SET or tubulin were imaged with a Licor scanner and band intensities quantified by laser scanning densitometry. $\mathrm{n}=4$ mice per group. $\mathrm{p}>0.05$. in SET levels, could account for the observed beneficial changes in inflammation, AD behaviors and pathologies. Based on these findings and reports that SET levels increase in $\mathrm{AD}$, we propose that SET is a therapeutic target for disease modification in AD. Our previous reports $[1,2,5,6,17]$ and these findings also suggest that SET antagonism leading to increased PP2A activity may be a useful approach to reducing pathology, reducing inflammation and improving behavioral performance in subjects with AD.

\section{Acknowledgement}

This work was supported by grants from the National Institutes of Health (USA).

References

1 Christensen DJ, Ohkubo N, Oddo J, Van Kanegan MJ, Neil J, Li F, Colton CA, Vitek MP: Apolipoprotein E and peptide mimetics modulate inflammation by binding the SET protein and activating protein phosphatase 2A. J Immunol 2011;186:2535-2542.

-2 Laskowitz DT, Song P, Wang H, Mace B, Sullivan PM, Vitek MP, Dawson HN: Traumatic brain injury exacerbates neurodegenerative pathology: improvement with an apolipoprotein E-based therapeutic. J Neurotrauma 2010;27:1983-1995. 
-3 Wilcock DM, Lewis MR, Van Nostrand WE, Davis J, Previti ML, Gharkholonarehe N, Vitek MP, Colton CA: Progression of amyloid pathology to Alzheimer's disease pathology in an amyloid precursor protein transgenic mouse model by removal of nitric oxide synthase 2. J Neurosci 2008;28:1537-1545.

-4 Corder EH, Saunders AM, Strittmatter WJ, Schemechel DE, Gaskell PC, Small GW, Roses AD, Haines JL, Pericak-Vance MA: Gene dose of apolipoprotein-E type 4 allele and the risk of Alzheimer's disease in late onset families. Science 1993;261:921-923.

5 Lynch JR, Tang W, Wang H, Vitek MP, Bennett ER, Sullivan PM, Warner DS, Laskowitz DT: APOE genotype and an ApoE-mimetic peptide modify the systemic and central nervous system inflammatory response. J Biol Chem 2003;278:48529-48533.

-6 Laskowitz DT, McKenna SE, Song P, Wang H, Durham L, Yeung N, Christensen D, Vitek MP: COG1410, a novel apolipoprotein Ebased peptide, improves functional recovery in a murine model of traumatic brain injury. J Neurotrauma 2007;24:1093-1107.

7 Wang X, Blanchard J, Kohlbrenner E, Clement N, Linden RM, Radu A, Grundke-Iqbal I, Iqbal K: The carboxy-terminal fragment of inhibitor-2 of protein phosphatase-2A induces Alzheimer disease pathology and cognitive impairment. FASEB J 2010;24:4420-4432.
8 Deters N, Ittner LM, Gotz J: Substrate-specific reduction of PP2A activity exaggerates tau pathology. Biochem Biophys Res Commun 2009;379:400-405.

-9 Davis J, Xu F, Deane R, Romanov G, Previti ML, Zeigler K, Zlokovic BV, Van Nostrand WE: Early-onset and robust cerebral microvascular accumulation of amyloid beta-protein in transgenic mice expressing low levels of a vasculotropic Dutch/Iowa mutant form of amyloid beta-protein precursor. J Biol Chem 2004;279:20296-20306.

10 Wink DA, Hines HB, Cheng RY, Switzer CH, Flores-Santana W, Vitek MP, Ridnour LA, Colton CA: Nitric oxide and redox mechanisms in the immune response. J Leukoc Biol 2011;89:873-891.

11 Oshima K, Uchikado H, Dickson DW: Perivascular neuritic dystrophy associated with cerebral amyloid angiopathy in Alzheimer's disease. Int J Clin Exp Pathol 2008;1:403408.

12 Vidal R, Calero M, Piccardo P, Farlow MR, Unverzagt FW, Méndez E, Jiménez-Huete A, Beavis R, Gallo G, Gomez-Tortosa E, Ghiso J, Hyman BT, Frangione B, Ghetti B: Senile dementia associated with amyloid beta protein angiopathy and tau perivascular pathology but not neuritic plaques in patients homozygous for the APOE-epsilon4 allele. Acta Neuropathol 2000;100:1-12.
13 Van Nostrand WE, Xu F, Rozemuller AJ, Colton CA: Enhanced capillary amyloid angiopathy-associated pathology in Tg-SwDI mice with deleted nitric oxide synthase 2 . Stroke 2010;41:S135-S138.

14 Livak KJ, Schmittgen TD: Analysis of relative gene expression data using real-time quantitative PCR and the 2(-Delta Delta C(T)) method. Methods 2001;25:402-408.

15 Sontag E, Nunbhakdi-Craig V, Sontag J-M, Diaz-Arrastia R, Ogris E, Dayal S, Lentz SR, Arning E, Bottiglieri T: Protein phosphatase 2A methyltransferase links homocysteine metabolism with tau and amyloid precursor protein regulation. J Neurosci 2007;27:27512759.

16 van Eersel J, Ke YD, Liu X, Delerue F, Kril JJ, Gotz J, Ittner LM: Sodium selenate mitigates tau pathology, neurodegeneration, and functional deficits in Alzheimer's disease models. Proc Natl Acad Sci USA 2010;107:1388813893.

17 Li FQ, Fowler KA, Neil JE, Colton CA, Vitek MP: An apolipoprotein E-mimetic stimulates axonal regeneration and remyelination after peripheral nerve injury. J Pharmacol Exp Ther 2010;334:106-115. 